\title{
Large-scale dynamics and polarities of magnetic structures
}

\author{
N. Meunier \\ Laboratoire d'Astrophysique de l'Observatoire Midi-Pyrénées, 57 avenue d'Azereix, BP 826, 65008 Tarbes Cedex, France \\ e-mail: meunier@bagn.obs-mip.fr
}

Received 23 November 2004 / Accepted 16 February 2005

\begin{abstract}
A large data set of MDI magnetograms is studied in order to analyse the dependence of the global dynamics of magnetic structures on their polarity. Two complementary techniques (feature tracking and correlation tracking) are used to determine the differential rotation and meridional circulation. A faster rotation of leading polarity structures is found for active areas, while a faster rotation of following polarity structures is found for weak field areas. However the latter is found only when using correlation tracking: individual small features do not seem to exhibit any difference between leading and following polarities. From correlation tracking, the mixed polarity areas (with a low activity level) do rotate as the following polarity areas. Furthermore, the latter have a more poleward motion when the correlation tracking is used.
\end{abstract}

Key words. Sun: magnetic fields - Sun: photosphere - Sun: faculae, plages

\section{Introduction}

A variation of the angular rotation velocity (hereafter rotation rates, in heliographic degrees per day) of plages and sunspots have been previously observed by several authors (see Howard 1996, for a review). However, such a variation with polarity for the magnetic network has not been obtained up to now, probably due to the limited amount of data (Komm et al. 1993a,b; Meunier 1999) or due to the lack of information about the polarities (Meunier et al. 1997). In fact, Meunier (1999) attempted that study, but the errorbars were too large to allow any significant result. Furthermore, the variation of the meridional circulation with polarity has not been studied as well, since this motion is of a much smaller amplitude (two orders of magnitude) and therefore requires an even better data set. The main work is that of Ward (1973), who studied the latitudinal motion of sunspots and its dependence on various characteristics, including polarities. He found a more poleward motion of spots with the following polarity.

This type of analysis is very important in order to understand the emergence of active regions and their decay. Analysis of the magnetic network dynamics in that context should also provide some clues about the decay of active regions into network structures.

The global dynamics (differential rotation and meridional circulation) over a very large data set of MDI magnetograms were studied by Meunier (2005a, hereafter Paper I). Here, I use this data set in order to study the variation of the dynamics with polarity. The data processing and analysis are briefly described in Sect. 2. Section 3 presents the results for differential rotation and Sect. 4 the results for the meridional circulation. The results are discussed in Sect. 5.

\section{Data and processing}

\subsection{MDI data}

The data set is a subset of the one used in Paper I for the study of the rotation rate and meridional circulation: I use a data set of 5-min averaged full-disk magnetograms obtained by MDI on SOHO (Scherrer et al. 1995) between June 1997 and April 2004, processed at the 1.8 level. These magnetograms are separated by $96 \mathrm{~min}$ or a multiple of $96 \mathrm{~min}$. The polarities of structures or regions (leading or following) will be determined considering that at a given time, all structures obey Hale's law, which is why the data set is slightly reduced compared to Paper I, and it ensures that no structure from the previous cycle is present. The butterfly diagram for these structures is shown in Fig. 1. For Cycle 23, the leading polarity was positive in the Northern hemisphere and negative in the Southern hemisphere.

The noise level in 5-min magnetograms is reduced compared to that of 1-min magnetograms and is of the order of $8 \mathrm{G}$. An additional source of error comes from the shutter noise, which induces a small random shift of the zero level in the magnetograms. This error has been studied in detail by Liu et al. (2003). One way to estimate this noise level is to compute the distribution of magnetic field values in the image and then to use a gaussian fit on this distribution to compute the offset. This determination will, however, be strongly influenced by the presence of activity, but because filtering this time series with many gaps is not straightforward, it is beyond the scope of this paper. We compared the results when no offset is performed or when a raw offset is applied for the first part of the series (560 days, with low activity level). The influence on the results 


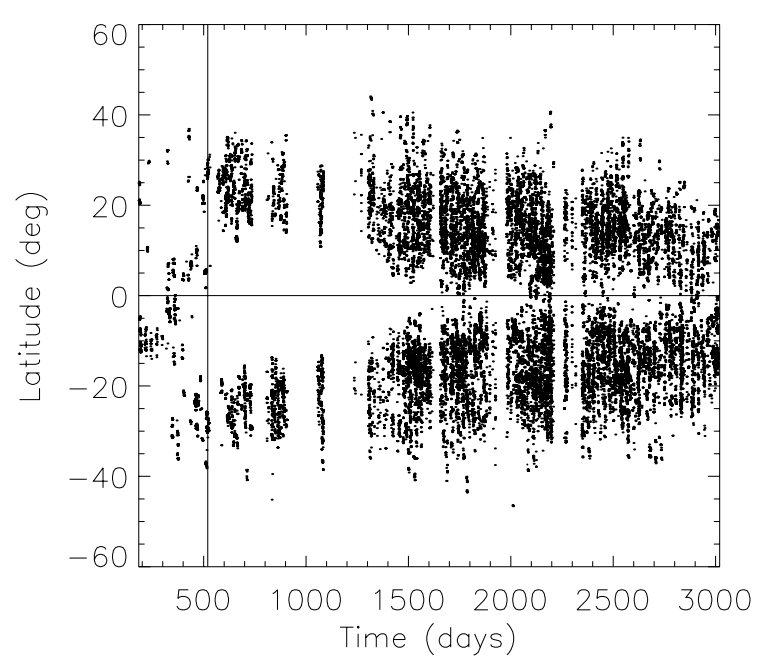

Fig. 1. Butterfly diagram of the structures larger than $500 \mathrm{Mm}^{2}$ for the whole data set. Day 1 corresponds to January 1, 1996. The vertical solid line at day 520 (June 1997) corresponds to the limit considered in this paper.

is much smaller than the errorbars, so that we consider that the effect can be neglected and the results presented in this paper are obtained with no offset correction.

\subsection{Data processing}

The data processing here is identical to that of Paper I. Both feature tracking (hereafter FT) and correlation tracking (hereafter CT) are used. I refer to Paper I for all details and here indicate only the most important points (see also Meunier 1999). Feature tracking is done by identifying magnetic structures above a threshold of $40 \mathrm{G}$ (separately for the 2 polarities), which is well above the noise, and then by following them on pairs of magnetograms (separated by 96 minutes). Correlation tracking is done by cross-correlating boxes of size $7.68 \times 7.68 \mathrm{deg}^{2}\left(93 \times 93 \mathrm{Mm}^{2}\right)$ on these same pairs of magnetograms after remapping. Various quantities such as the magnetic field, maximum magnetic field, flux, and size of the structures are computed. Some relevant correspondences between variables are discussed in Sect. 2.3 (see also Paper I).

Note that there are more negative polarity boxes/structures than positive polarity. Because this is seen in both hemispheres (already noted in Meunier 2003) it will not change the results reported here qualitatively; however, if that is an instrumental bias, it could in theory create artificial asymmetries between hemispheres, such as a difference between polarities larger in one hemisphere compared to the other.

\subsection{Correspondence between sizes and magnetic fields}

The polarity of FT structures is easily defined since for one structure the polarity of all pixels is the same. The correspondences between the size $A_{t}$, magnetic flux $\Phi_{t}$, and the maximum of the absolute magnetic field $B m_{t}$ are shown in Paper I. It is important to keep in mind that $B m_{t}$ in structures larger than
$500 \mathrm{Mm}^{2}$ may be saturating due to a bias in MDI data. Except for this limitation, the non-linearity studied by Berger \& Lites (2003) does not affect the results presented here.

Polarity in CT boxes is less straightforward, because in mixed polarity regions there are magnetic features of both polarities inside a given box. To determine the polarity in a given box, I compute the average (signed) magnetic field $\left\langle B_{\mathrm{c}}\right\rangle$ in that box. Roughly, $\left\langle B_{\mathrm{c}}\right\rangle \sim 0$ means a mixed polarity area, and $\left\langle B_{\mathrm{c}}\right\rangle$ close to $\left\langle\left|B_{\mathrm{c}}\right|\right\rangle$ means a unipolar area with polarity of one sign or the other. As a consequence, the significance of $\left\langle B_{\mathrm{c}}\right\rangle$ depends on the activity level as well, and it will be useful in the following to use the variable $\left\langle B_{\mathrm{c}}\right\rangle /\left\langle\left|B_{\mathrm{c}}\right|\right\rangle$. This is illustrated in Fig. 2. The variation in behavior of $\left\langle B_{\mathrm{c}}\right\rangle$ for various $\left\langle\left|B_{\mathrm{c}}\right|\right\rangle$ domains is also illustrated in Fig. 3. In the range 9-12 G, for example, the 3 components are clearly visible with the mixed polarity component centered on 0 , and the 2 unipolar components centered on $\pm 6 \mathrm{G}$.

Because of the noise level, $\left\langle\left|B_{\mathrm{c}}\right|\right\rangle$ is slightly overestimated, and the effect is larger for weak magnetic regions; $\left\langle B_{\mathrm{c}}\right\rangle$ is not affected significantly however. As a consequence, the ratios $\left\langle B_{\mathrm{c}}\right\rangle /\left\langle\left|B_{\mathrm{c}}\right|\right\rangle$ are also slightly underestimated. Note that $\left\langle B_{\mathrm{c}}\right\rangle /\left\langle\left|B_{\mathrm{c}}\right|\right\rangle$ close to \pm 1 are usually for very active areas.

\section{Rotation rates}

\subsection{Correlation tracking}

Figure 4 shows the variation of the $\mathrm{CT}$ residual rotation rates versus $\left\langle B_{\mathrm{c}}\right\rangle$ separately for the two hemispheres. It shows that for $\left|\left\langle B_{\mathrm{c}}\right\rangle\right|$ larger than $20 \mathrm{G}$, the areas of following polarity are rotating slower than that of leading polarity. Below that threshold, the opposite is observed, as the areas of following polarity are rotating faster. Note that here small $\left\langle B_{\mathrm{c}}\right\rangle$ zones correspond either to areas of mixed polarity with a large active level or to all kinds of weak field regions.

The plot of these residuals versus $\left\langle B_{\mathrm{c}}\right\rangle /\left\langle\left|B_{\mathrm{c}}\right|\right\rangle$ (Fig. 5) confirms that for weak fields the following polarity is rotating faster. In fact, on that plot, the strong activity levels are present for all ratios, and because there are many more weak field areas, they are dominating the data set. This is why this plot does not show the fastest rotation of leading polarities for strong field regions. One should also note that the plots are not symmetrical with respect to $\left\langle B_{\mathrm{c}}\right\rangle=0$. For example in the Northern hemisphere, the curve is quite flat for all small positive ratios, but much steeper for a negative ratio. Another way to see it is that the leading polarity areas exhibit a quite constant rotation rate residual over the whole range of ratios, while the following polarity areas exhibit strong variation, with a slower rotation rate for mixed polarity areas as well as unipolar regions. Intermediate areas rotate faster by $0.05 \mathrm{deg} / \mathrm{day}(7 \mathrm{~m} / \mathrm{s}$ at the equator).

To better understand the mixing of $\left\langle\left|B_{\mathrm{c}}\right|\right\rangle$ and $\left\langle B_{\mathrm{c}}\right\rangle /\left\langle\left|B_{\mathrm{c}}\right|\right\rangle$, Fig. 5 also shows the variation of $\left\langle\left|B_{\mathrm{c}}\right|\right\rangle$ with $\left\langle B_{\mathrm{c}}\right\rangle /\left\langle\left|B_{\mathrm{c}}\right|\right\rangle$. The pattern seen at the top of Fig. 5 is mostly present up to $N$ pix around 600 , that is, a $15 \%$ coverage of the boxes by fields above $40 \mathrm{G}$. This corresponds well to the $20 \mathrm{G}$ level (see Paper I, Fig. 3). Note that the following polarity residuals are maximum 

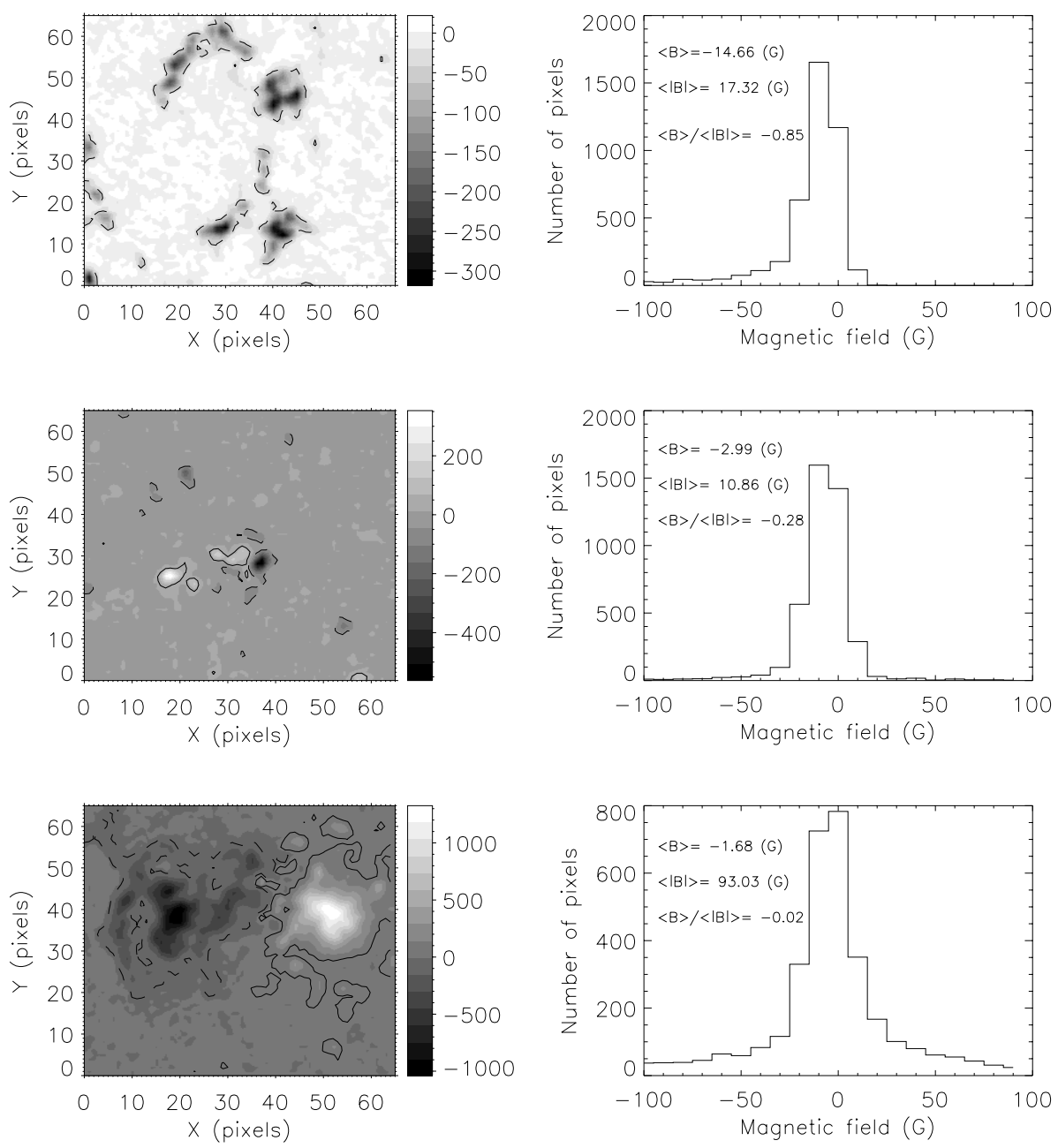

Fig. 2. Left: 3 examples of CT boxes, with the $40 \mathrm{G}$ level outlined (threshold used in the FT analysis), in solid lines for positive magnetic fields, and dashed lines for negative magnetic fields. Right: distribution of the corresponding magnetic field values in these areas, with an indication of $\left\langle B_{\mathrm{c}}\right\rangle,\left\langle\left|B_{\mathrm{c}}\right|\right\rangle,\left\langle B_{\mathrm{c}}\right\rangle /\left\langle\left|B_{\mathrm{c}}\right|\right\rangle$.

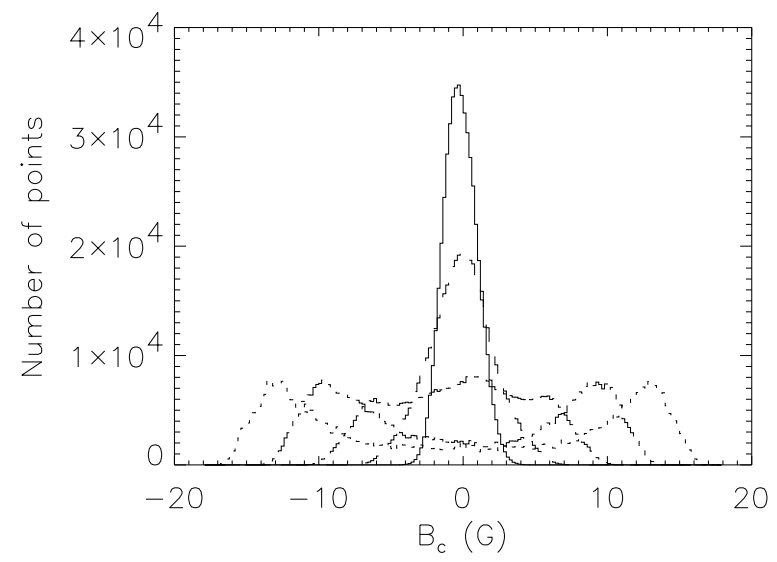

Fig. 3. Distribution of $\left\langle B_{\mathrm{c}}\right\rangle$ for $\left\langle\left|B_{\mathrm{c}}\right|\right\rangle$ in the range 3-6 G (solid), 6-9 G (dashed), 9-12 G (dotted-dashed), 12-15 G (dotted).

for $\left|\left\langle B_{\mathrm{c}}\right\rangle /\left\langle\left|B_{\mathrm{c}}\right|\right\rangle\right| \sim 0.5$, which is also where the distribution of the number of points is changing its slope (bottom of Fig. 5).

Figure 6 shows the variation of residual rotation rates for the two polarities and the two hemispheres separately, as a function of $\left\langle\left|B_{\mathrm{c}}\right|\right\rangle$ and $B m_{\mathrm{c}}$. For $\left\langle\left|B_{\mathrm{c}}\right|\right\rangle$ above $\sim 20 \mathrm{G}$ and $B m_{\mathrm{c}}$ above $\sim 500 \mathrm{G}$, areas of leading polarity are again rotating faster than areas of following polarity as in Fig. 4. Below, it is the opposite again and is quite significant over a wide range of activity levels. For very small $\left\langle\left|B_{\mathrm{c}}\right|\right\rangle$, the difference may disappear, however. In order to make a better link with Fig. 4 , Fig. 7 shows the residual rotation rates versus latitude for one of the domains displayed in Fig. 3, i.e. 9-12 G. Each curve represents one of the 3 components. It confirms previous results concerning the rotation of areas of following and leading polarity in weak field regions. It also shows that areas of mixed polarity are rotating at a rate similar to that of the following polarity. There is, however, a strong asymmetry between hemispheres at high latitudes.

\subsection{Feature tracking}

Figure 8 shows variation in the residual rotation rates versus feature size, magnetic flux, and maximum magnetic fields, and does so separately for the two polarities and the two hemispheres for the FT method. At large scales, features with the leading polarity are rotating faster than those with the 


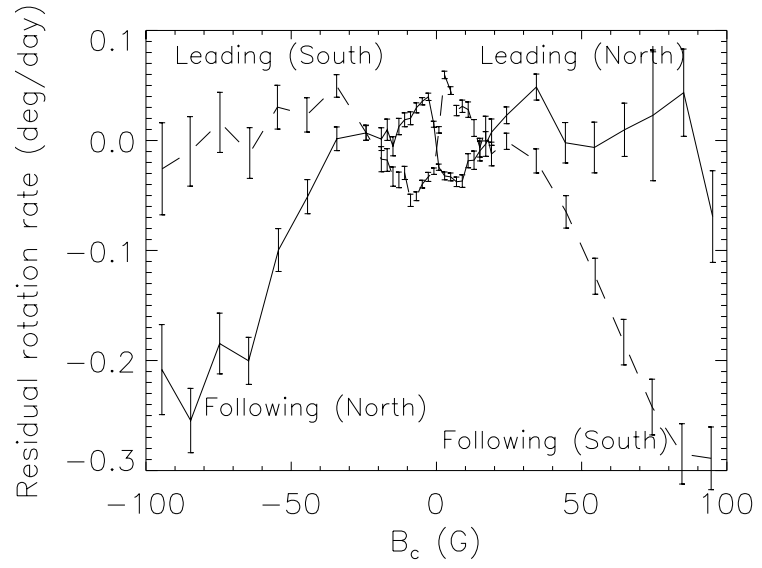

Fig. 4. CT residual rotation rates versus $\left\langle B_{\mathrm{c}}\right\rangle$ for the Northern hemisphere (solid line) and Southern hemisphere (dashed line).
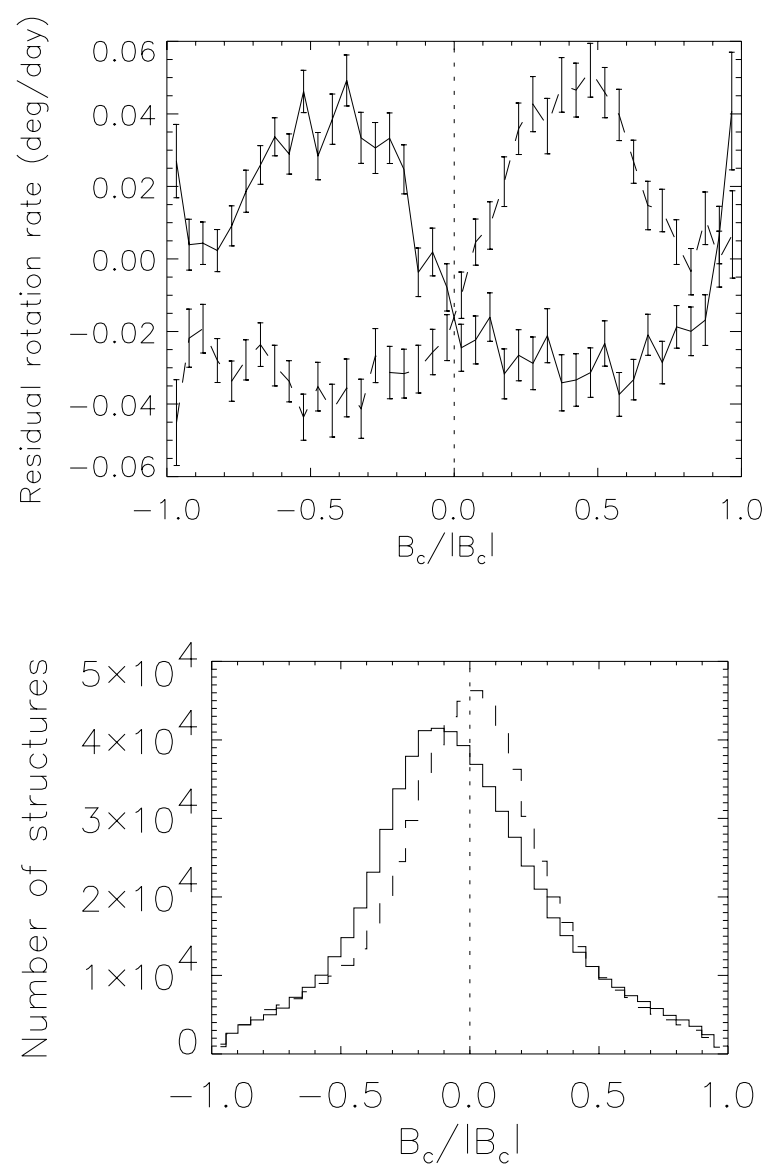

Fig. 5. Top: CT residual rotation rates versus $\left\langle B_{\mathrm{c}}\right\rangle /\left\langle\left|B_{\mathrm{c}}\right|\right\rangle$ for the Northern hemisphere (solid line) and Southern hemisphere (dashed line). Bottom: same for the number of boxes versus $\left\langle B_{\mathrm{c}}\right\rangle /\left\langle\left|B_{\mathrm{c}}\right|\right\rangle$.

following polarity, similar to what is observed with the CT method in Fig. 6.

However, below this threshold of a few hundred $\mathrm{Mm}^{2}$ (as well as a flux of $\sim 2 \times 10^{20} \mathrm{Mx}$, and a maximum field of $\sim 400 \mathrm{G}$ ), no difference between structures of following and leading polarities is observed, at the level of our errorbars (down to $1 \mathrm{~m} / \mathrm{s}$ in Table 1), as is seen with the CT method. Furthermore, if one selects boxes in the range 9-12 G for example (for which the difference was quite large), and then studies the dynamics of the FT structures present inside these boxes, no reversal is seen either, as shown in Table 1. An attempt was made to identify small bipoles, which led to very few structures; and they did not exhibit a different behavior between leading and following polarities either.

\section{Meridional circulation}

\subsection{Correlation tracking}

As for Figs. 4 and 5, Fig. 9 exhibits the residual meridional circulation versus $\left\langle B_{\mathrm{c}}\right\rangle$ and $\left\langle B_{\mathrm{c}}\right\rangle /\left\langle\left|B_{\mathrm{c}}\right|\right\rangle$ separately for the two hemispheres. Remember that a positive residual means a more poleward motion than the average in that latitude band. These two plots show several important characteristics. On the first plot, in both hemispheres areas with following polarity have a more poleward motion than areas with leading polarity. This is mostly seen on the plot versus $\left\langle B_{\mathrm{c}}\right\rangle$. The plot versus $\left\langle B_{\mathrm{c}}\right\rangle /\left\langle\left|B_{\mathrm{c}}\right|\right\rangle$ shows that mixed polarity regions move more poleward than the unipolar areas, which is probably mostly the case for weak field areas only, because the corresponding peak on the plot versus $\left\langle B_{\mathrm{c}}\right\rangle$ is very narrow (a few Gauss). This is also consistent with the larger poleward meridional circulation observed for weaker fields in Paper I. As for the rotation rate plot in the previous section, we observe on the plot versus $\left\langle B_{\mathrm{c}}\right\rangle /\left\langle\left|B_{\mathrm{c}}\right|\right\rangle$ an asymmetry close to mixed polarity areas (ratio in the range -0.2 to 0.2 ) again with the following polarity area moving more poleward, but only for the Southern hemisphere.

In Fig. 10, the residual meridional circulation versus $\left\langle\left|B_{\mathrm{c}}\right|\right\rangle$ and $B m_{\mathrm{c}}$ shows that for strong field regions, the area of following polarity are moving more poleward, which is true in both hemispheres down to $\left\langle\left|B_{\mathrm{c}}\right|\right\rangle \sim 20 \mathrm{G}$ and $B m_{\mathrm{c}} \sim 600 \mathrm{G}$. Below that it is more complex, as the following part also seems to be moving more poleward for weak field areas (as in Fig. 9, bottom), but only in the Southern hemisphere. It is the opposite in the Northern hemisphere.

Figure 11 shows the residual meridional circulation versus latitude for one of the domains displayed in Fig. 3, i.e. 9-12 G. Each curve represents one of the 3 components, as in Fig. 7 for the residual rotation rate. For latitudes smaller than $20^{\circ}$, the mixed polarity areas tend to move more poleward than the others, and areas of leading polarity move more poleward than areas of following polarity. At latitude larger than $20^{\circ}$, the mixed polarity areas move less poleward than the others, while the areas of following polarity move more poleward. This dependence on latitude may explain why we do observe some differences between Figs. 9 and 10. There are also some differences between the two hemispheres, showing that the meridional circulation is far from symmetric between them.

\subsection{Feature tracking}

Similar plots for the FT residual meridional circulation are not shown here, and no difference between the polarities is seen for any size range. Here again the CT and FT behavior is very different, even for large structures. 

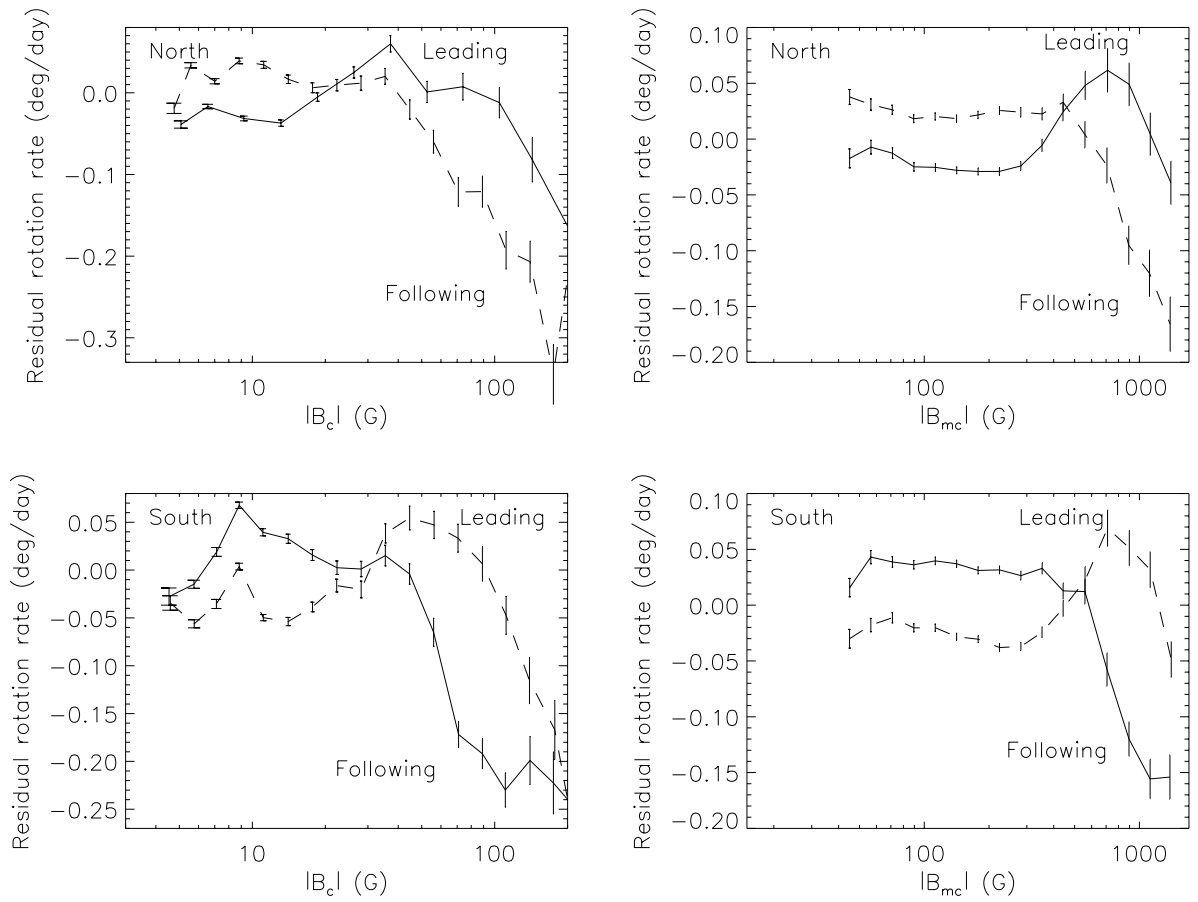

Fig. 6. CT residual rotation rates versus $\left\langle\left|B_{\mathrm{c}}\right|\right\rangle($ Left $)$ and versus $B m_{\mathrm{c}}$ (Right) for the positive polarity (solid line, leading in the Northern hemisphere) and negative polarity (dashed line, following in the Northern hemisphere). Top: Northern hemisphere. Bottom: Southern hemisphere.

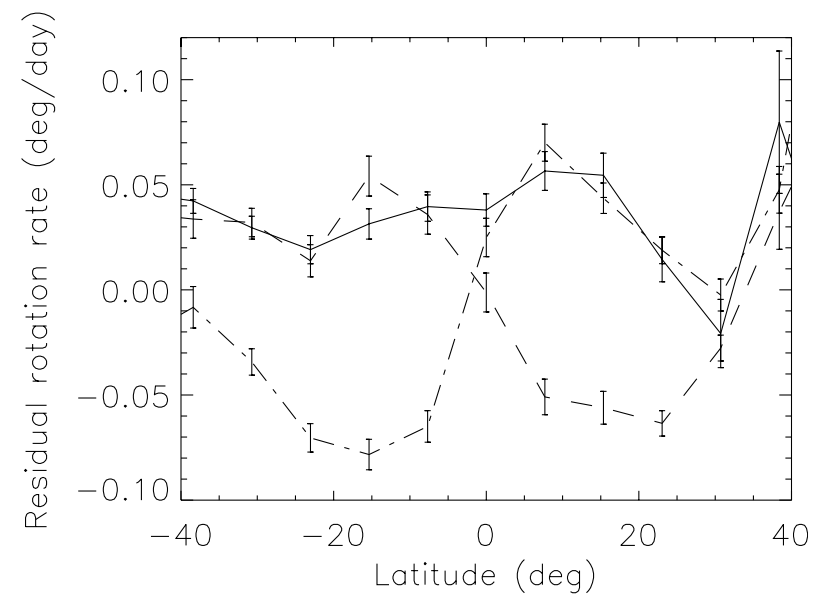

Fig. 7. CT residual rotation rates for $\left\langle\left|B_{\mathrm{c}}\right|\right\rangle$ in the range $9-12 \mathrm{G}$. The solid curve represents mixed polarity areas $\left(\left\langle B_{\mathrm{c}}\right\rangle\right.$ between -4 and $\left.4 \mathrm{G}\right)$, the dotted-dashed line represents $\left\langle B_{\mathrm{c}}\right\rangle$ smaller than $-4 \mathrm{G}$ and the dashed line represents $\left\langle B_{\mathrm{c}}\right\rangle$ larger than $4 \mathrm{G}$.

\section{Discussion and conclusion}

In this Paper I have studied the dynamics of magnetic features down to network-size structures depending on their polarity in each hemisphere. Two complementary methods (CT and FT) have been used. The most significant result is that for $\langle|B \mathrm{c}|\rangle$ below 20-30 G (Bm below 400-500 G), areas (defined with CT) of the following polarity are rotating faster than those of leading polarity, which is the opposite of what is observed in active regions. We also find that the positive gradient of the residual rotation rate versus $\left\langle\left|B_{\mathrm{c}}\right|\right\rangle$ or $B m_{\mathrm{c}}$ observed in Paper I for $\left\langle\left|B_{\mathrm{c}}\right|\right\rangle$ below $10 \mathrm{G}$ is in fact occuring at different values of $\left\langle\left|B_{\mathrm{c}}\right|\right\rangle$ for the two polarities, which contributes strongly to the observed reversal. It is in the range $\left\langle\left|B_{\mathrm{c}}\right|\right\rangle$ below $10 \mathrm{G}$ for areas of following polarity, but in the range $15-40 \mathrm{G}$ for areas of leading polarity. For $B m_{\mathrm{c}}$, we mostly see a positive gradient for leading polarities in the range 300-700 G. I observe that this reversal, observed for weak field regions, is present even for small $\left\langle B_{\mathrm{c}}\right\rangle /\left\langle\left|B_{\mathrm{c}}\right|\right\rangle$ ones (i.e. regions with a small degree of unipolarity), while mixed polarity areas are rotating like areas of following polarity, although this reversal is not observed with FT.

Zhao et al. (2004) have observed a faster surface plasma rotation rate (deduced from a time-distance analysis) where following polarity was present, which is interpreted as a preferential location of these following polarity magnetic features in the following edge of supergranules. However, it is likely that magnetic features observed at the solar surface do not move following this supergranular flow because I do not observe it with FT despite the fact that the errorbars are smaller than their signal. Because it is observed only for CT, it is likely that whole pattern of a given polarity moves with this characteristic. We find a maximum difference of approximately $7 \mathrm{~m} / \mathrm{s}$ while Zhao et al. (2004) find a difference reaching 10 to $40 \mathrm{~m} / \mathrm{s}$ around $300 \mathrm{G}$, which is significantly larger. Therefore it could have the same origin (following polarities preferentially at the leading edge of supergranules) but with smaller amplitude due to a deeper anchoring, where we expect the horizontal flow to be smaller. It may also be related to how coronal holes are preferentially present in unipolar areas of following polarity (they may be rotating more rigidly, Timothy et al. 1975), but it is unlikely to be due to this only because the faster rotation rate of following polarity areas is also seen for boxes with a low degree of unipolarity. 

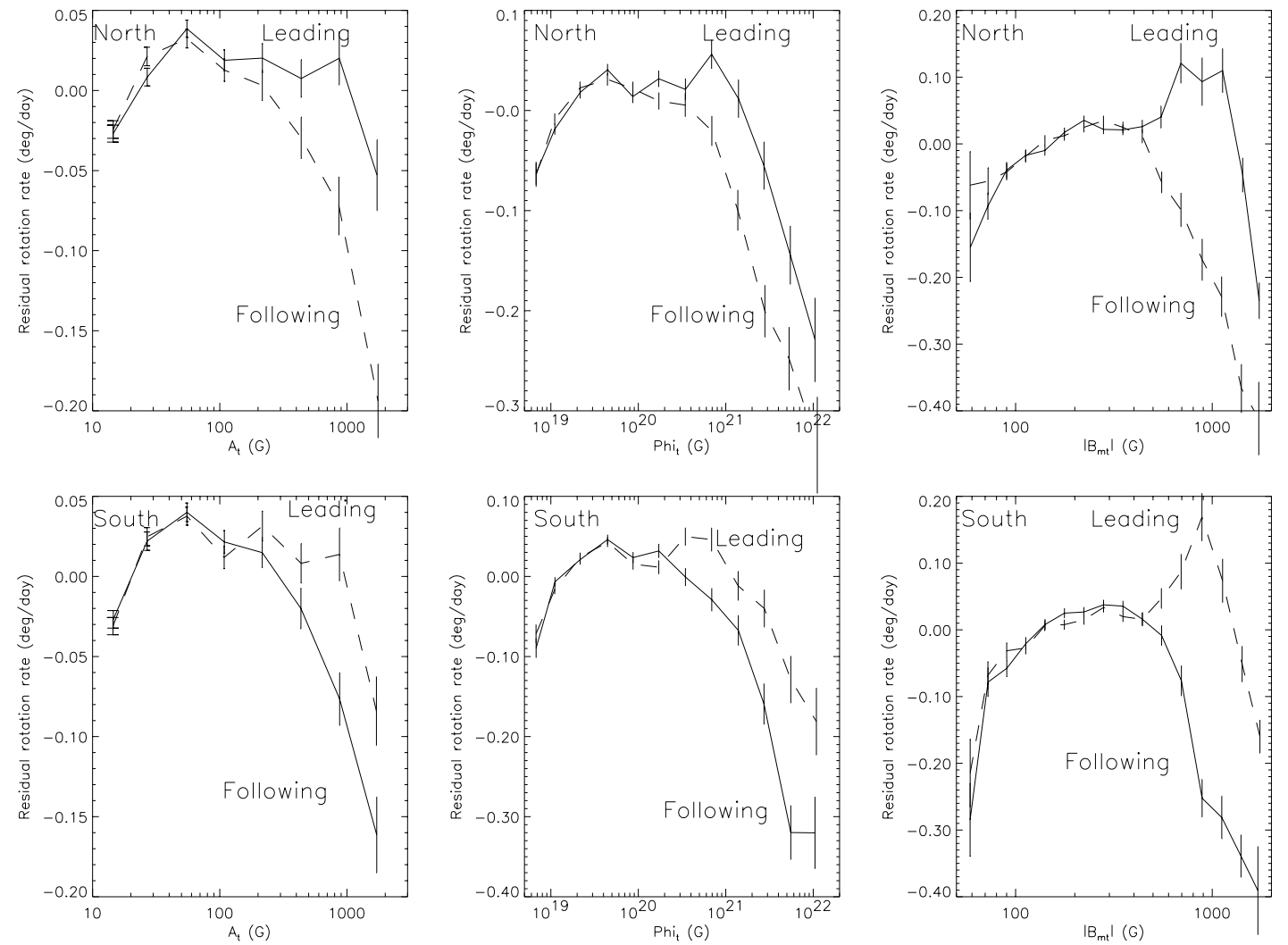

Fig. 8. FT residual rotation rates versus $A$ (left), versus $\Phi_{t}$ (middle), and versus $B m_{t}(l e f t)$ for the positive polarity (solid line, leading in the Northern hemisphere) and negative polarity (dashed line, following in the Northern hemisphere). Top: Northern hemisphere. Bottom: Southern hemisphere.
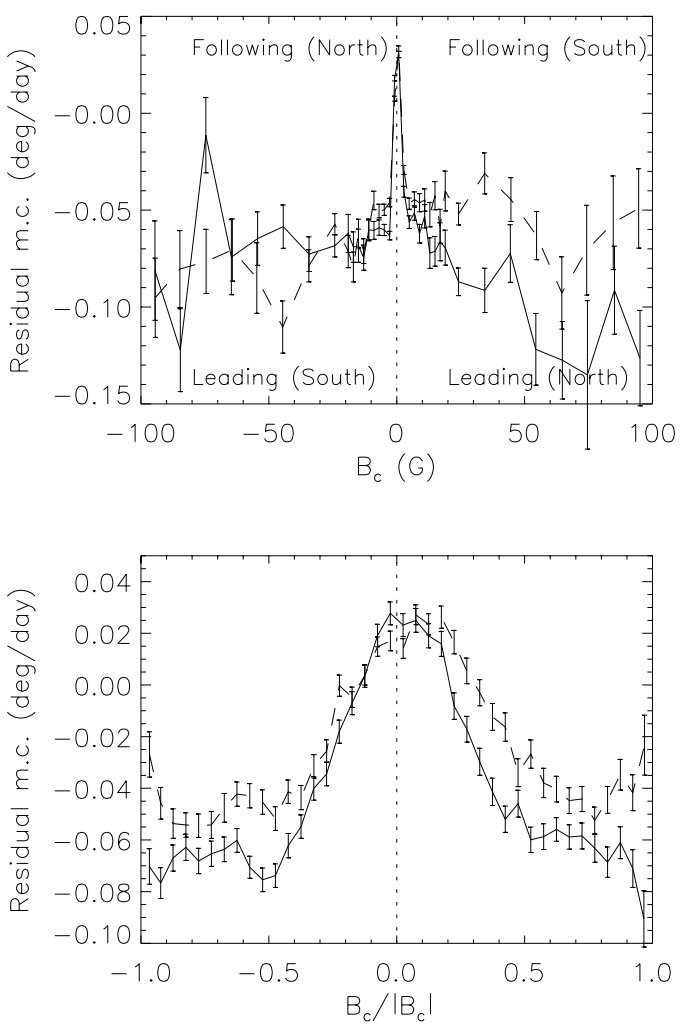

Fig. 9. Top: CT residual meridional circulation versus $\left\langle B_{\mathrm{c}}\right\rangle$ for the Northern hemisphere (solid line) and Southern hemisphere (dashed line). Bottom: same versus $\left\langle B_{\mathrm{c}}\right\rangle /\left\langle\left|B_{\mathrm{c}}\right|\right\rangle$.
Table 1. Residual rotation rates for the CT method (for $\left\langle\left|B_{\mathrm{c}}\right|\right\rangle$ in the range 9-12 G, when some FT structures are present) and for the FT method (for structures present in CT boxes), in deg/day.

\begin{tabular}{ccc}
\hline \hline Polarity & CT & FT \\
\hline Leading N & $-0.013 \pm 0.003$ & $0.015 \pm 0.008$ \\
Following N & $0.046 \pm 0.003$ & $0.015 \pm 0.008$ \\
Leading S & $-0.017 \pm 0.003$ & $0.016 \pm 0.008$ \\
Following S & $0.038 \pm 0.003$ & $0.026 \pm 0.008$ \\
\hline
\end{tabular}

Furthermore, a larger poleward meridional circulation is observed for areas of following polarities mostly for large structures, which is a new result, observed with CT only. It is similar to the result of Ward (1973) for sunspots, however, who found a more poleward motion of sunspots reaching $0.05 \mathrm{deg} / \mathrm{day}$ in the latitude range $15-20^{\circ}$. For weak field regions, we do observe a more poleward meridional circulation for areas of following polarities, but only in the Southern hemisphere (when averaged over all latitudes) and above $20^{\circ}$ in the Northern hemisphere. Following polarity areas with latitude in the range $0-20^{\circ}$ are moving less poleward. A more poleward meridional circulation for following polarities may reinforce the reversal of polar magnetic fields due to a diffusion towards the poles of these following polarities. However, the polar magnetic field reversal occurred first for the Northern hemisphere and only 1 year later in the Southern hemisphere 

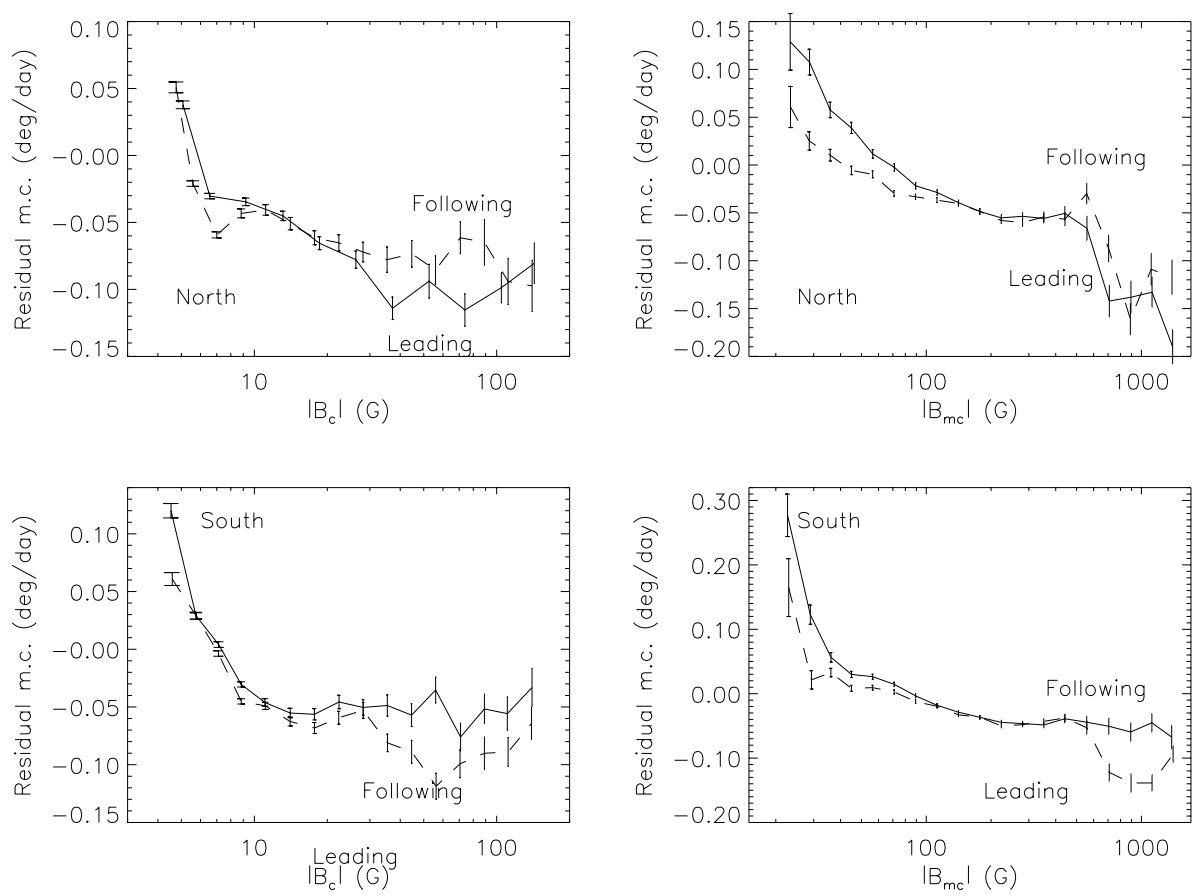

Fig. 10. CT residual meridional circulation versus $\left\langle\left|B_{\mathrm{c}}\right|\right\rangle$ (left) and versus $\left|B m_{\mathrm{c}}\right|$ (right) for the positive polarity (solid line, leading in the Northern hemisphere) and negative polarity (dashed line, following in the Northern hemisphere). Top: Northern hemisphere. Bottom: Southern hemisphere.

(Harvey \& Recely 2002; Wang et al. 2002), as was explained by Dikpati et al. (2004) by the reversed cell in meridional flow in the Northern hemisphere. One should note that a difference of $0.02 \mathrm{deg} / \mathrm{day}$ out of a meridional circulation of $0.2 \mathrm{deg} / \mathrm{day}$ over 60 degrees in latitude (from latitude $40^{\circ}$ to the poles) corresponds to a time-scale of 1 month only, i.e. much smaller than 1 year, so this factor is probably neglegible compared to other effects. Areas of mixed polarity with a weak field are also moving more poleward below $20^{\circ}$ latitude in both hemispheres and less poleward above. As has been shown using other approaches (Haber et al. 2002; Zhao \& Kosovichev 2004; Komm et al. 2004), the meridional circulation exhibits strong asymmetry between hemispheres.

The way the flux disappears in mixed polarity areas is not completely understood (Zwaan 1987). If the mixed polarities areas corresponded to regions at the interface between unipolar areas, we might expect the rotation rate of mixed polarity areas to be close to the average of following and leading polarity area rotation rates, which is not what we observe. This is also true for the meridional circulation. This does not lead to a direct conclusion about the way the flux evolves in the various contexts (unipolar, mixed) and disappear, but it provides a clue.

A faster rotation of sunspots with leading polarity has been observed before (see for example Gilman \& Howard 1985; Howard 1992a,c). However, Howard (1992c) finds that this is observed for growing spot groups only, while a reversal is observed for decaying regions. Howard (1996) also finds that plages with leading and following polarities are rotating at the same rate close to the equator, but that following polarity plages are rotating faster than the leading part at higher latitudes (the difference reaches $0.3 \mathrm{deg} / \mathrm{day}$ at $40^{\circ}$ latitude). His interpretation is as follows: during the growing phase, the emerging loop intersects the surface in two locations, leading to two regions of opposite polarities that are moving apart, providing a faster rotation rate for the leading polarity than for the following polarity. They suggest that during the decay phase, the two parts of the loop become closer again, leading to the reversal. It is not yet clear whether this is what is happening, but it would not apply directly to network features. Howard (1996) argues that the asymmetries between the leading and following parts of plages associated to the differential rotation elongating the features could create the faster observed rotation rate of following plages. However, if the faster rotation rate of the network region with the following polarity is the remnant of what is observed for plages, it is likely that the results obtained for plages are real.

A possibility for the faster rotation rate of following polarity areas could be a different orientation of the corresponding flux tubes (between leading and following). Indeed several authors have published some variations in the characteristics of these features (van Driel-Gesztelyi \& Petrovay 1990; Howard 1991, 1992b; and Meunier 2003, for network features), which could lead to some biases in the position of the features (we measure the line-of-sight magnetic field only). However, such a bias would lead to a variation of the rotation rate using FT as well, and not only for CT. It is still possible however that a different inclination would lead to a different interaction with supergranulation.

Another possibility could be that following polarity flux tubes are anchored deeper in the convection zone (in the shear layer, $7 \mathrm{~m} / \mathrm{s}$ represent $2 \mathrm{Mm}$ ). This interpretation has to be taken with caution, however, because Paper I shows that the rotation 


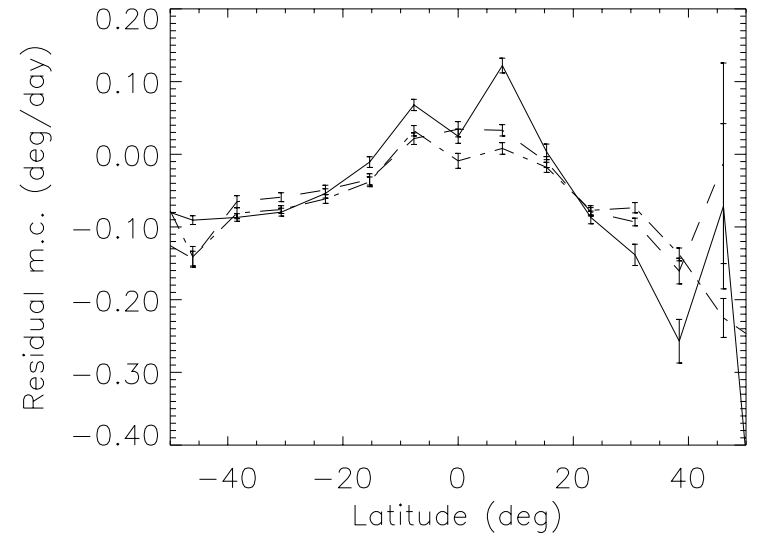

Fig. 11. CT residual meridional circulation for $\left\langle\left|B_{\mathrm{c}}\right|\right\rangle$ in the range 9-12 G. The solid curve represents mixed polarity areas $\left(\left\langle B_{\mathrm{c}}\right\rangle\right.$ between -4 and $4 \mathrm{G}$ ); the dotted-dashed line represents $\left\langle B_{\mathrm{c}}\right\rangle$ smaller than $-4 \mathrm{G}$; and the dashed line represents $\left\langle B_{\mathrm{c}}\right\rangle$ larger than $4 \mathrm{G}$.

rates of magnetic features cannot be interpreted only by their anchoring in certain layers. For example, because the following part of active region usually decays faster than the leading part, and following flux tubes may have more time to be swept deeper. This would also be consistent with the results found in Paper I where small features rotate faster when they are far from a large structure. Since they probably result from the decay of these regions, their faster rotation rate may result from submergence of these fields to a deeper region before appearing again as network features with faster rotation rates. On the other hand, this is not completely consistent with a more poleward motion since the meridional circulation seems to decrease inward (Haber et al. 2002; Komm et al. 2004; Zhao \& Kosovichev 2004). It is also possible that the action of the supergranular flow on the flux tubes of either the following or the leading polarity is different if they have a different inclination. However, here again the supergranular flow should act on the pattern formed by the network features, and not the features themselves, since FT does not show any difference between following and leading polarities.

The reason for the faster rotation rate and more poleward motion of following polarity areas with weak field is therefore not clear yet. Future work on the temporal variations may help to understand it better. In a future work, we will study these temporal variations (Meunier 2005b) and compare them to the results of Zhao et al. (2004). Their relation with possible largescale patterns in longitude and with coronal holes will also be the subject of future work: these patterns could be associated to large-scale zonal flows.

Acknowledgements. SOHO is a mission of international cooperation between the European Space Agency (ESA) and NASA. I thank Y. Liu, R. Bogart, H. Carfantan and the referee for useful comments on MDI data and this work.

\section{References}

Berger, T., \& Lites, A. 2003, Sol. Phys., 213, 213

Dikpati, M., de Toma, G., Gilman, P. A., Arge, C. N., \& White, O. R. 2004, ApJ, 601, 1136

Gilman, P. A., \& Howard, R. 1985, ApJ, 295, 233

Haber, D. A., Hindman, B. W., Toomre, J., et al. 2002, ApJ, 570, 855

Harvey, K. L., \& Recely, F. 2002, Sol. Phys., 211, 31

Howard, R. F. 1991, Sol. Phys., 134, 233

Howard, R. F. 1996, ARA\&A, 34, 75

Howard, R. F. 1992a, Sol. Phys., 142, 47

Howard, R. F. 1992b, Sol. Phys., 137, 205

Howard, R. F. 1992c, Sol. Phys., 137, 51

Howard, R. F., \& Stanchfield, D. H. II 1995, Sol. Phys., 156, 29

Komm, R. W., Howard, R. F., \& Harvey, J. W. 1993a, Sol. Phys., 145, 1

Komm, R. W., Howard, R. F., \& Harvey, J. W. 1993b, Sol. Phys., 147, 203

Komm, R. W., Corbard, T., Durney, B. R., et al. 2004, ApJ, 605, 554

Liu, Y., Xuepu, Z., \& Hoeksema, J. T. 2003, Sol. Phys., 219, 39

Meunier, N. 1999, ApJ, 527, 967

Meunier, N. 2003, A\&A, 405, 1107

Meunier, N. 2005a, A\&A, 436, 1075 (Paper I)

Meunier, N. 2005b, A\&A, submitted

Meunier, N., Nesme-Ribes, E., \& Grosso, N. 1997, A\&A, 319, 673

Scherrer, P., Bogart, R. S., Bush, R. I., et al. 1995, Sol. Phys., 162, 129

Timothy, A. F., Krieger, A. S., \& Vaiana, G. S. 1975, Sol. Phys., 42, 135

van Driel-Gesztelyi, L., \& Petrovay, K. 1990, Sol. Phys., 126, 285

Wang, Y.-M., Sheeley, N. R. Jr., \& Andrews, M. D. 2002, J. Geophys. Res., 107, 1465

Ward, F. 1973, Sol. Phys., 527

Zhao, J., Kosovichev, A. G., \& Duvall, T. L. Jr. 2004, ApJ, 607, L135

Zhao, J., \& Kosovichev, A. G. 2004, ApJ, 603, 776

Zwaan, C. 1987, ARA\&A, 25, 83 